\title{
Proton induced modification in makrofol-DE
}

\author{
N L SINGH*, NILAM SHAH and K P SINGH ${ }^{\dagger}$ \\ Physics Department, M.S. University of Baroda, Vadodara 390 002, India \\ ${ }^{\dagger}$ Department of Physics, Punjab University, Chandigarh 160 014, India
}

MS received 25 February 2005; revised 19 April 2005

\begin{abstract}
Irradiation effects of a $3 \mathrm{MeV}$ proton beam on polycarbonate (makrofol-DE (MFD)) have been studied with respect to its electrical, thermal and structural behaviour by using an LCR meter, DSC/TGA and FTIR spectroscopy. The dielectric loss/constant was observed to change with the fluence. Thermal analysis revealed that chain scission is the dominant phenomena in irradiated samples based on the reduction of its thermal stability by about $19 \%$ at a fluence of $10^{15}$ ions $/ \mathrm{cm}^{2}$, which is also corroborated by FTIR spectra. No significant change in intensity of the absorbance bands of the irradiated sample was observed up to a fluence of $10^{14}$ ions $/ \mathrm{cm}^{2}$ while on increasing fluence $\left(10^{15}\right.$ ions $\left./ \mathrm{cm}^{2}\right)$ the polymer structure was modified. It appears from DSC thermograms that $T_{\mathrm{g}}$ is observed to change with fluence.
\end{abstract}

Keywords. Makrofol-DE; FTIR; DSC; TGA; a.c. electrical frequency.

\section{Introduction}

Polycarbonate (MFD) is an amorphous polymer with attractive engineering properties including high impact strength, low moisture absorption, low combustibility, good dimensional stability and high light transmittance. The latter property has resulted in the application of PC as an impact-resistance substitute for window glass. PC particle track-etched membranes are used as templates in nano-tubes and nano-wires manufacturing (Piraux et al 1999; Jerome et al 2000). Inspite of this intensive use of PC in the nuclear membrane field, chemical modifications induced by ion beam irradiation has not received much attention (Dehaye et al 2003). Irradiation of polymers results in chemical and structural changes which lead to an alteration of their properties. Both cross-linking and degradation take place during proton irradiation and are closely related to irradiation fluences. At a certain fluence, cross-linking becomes predominant and mechanical properties are improved (Venkatesan et al 1987).

The effect of ion beam irradiation on PC has already been reported. The chemical modifications are induced by swift heavy ions (SHI) on a model compound of PC, i.e. diphenyl carbonate (DPC) (Ferain and Legras 1993). They have established the similarity between heavy ion irradiation and heat treatment. On the basis of these results, they explained the preferential chemical attack along the tracks of the irradiated film. The degradation processes in PC, PET, PI and PS were studied by SHI irradiations inducing electronic stopping power higher than 4.0 $\mathrm{MeV} \mathrm{mg}^{-1} \mathrm{~cm}^{2}$ (Steckenreiter et al 1999). They

\footnotetext{
*Author for correspondence (singhnl_msu@yahoo.com)
}

observed alkyne formation in all irradiated samples using in situ Fourier transform infrared (FTIR) spectroscopy. Electron spin resonance (ESR) investigations of SHI irradiated PC was performed to study the nature of free radicals as well as exchange interactions among them on the basis of track structure (Chipara and Reyes-Romero 2002). Chemical changes in PC induced by very high energetic ions (> GeV) were studied using ex situ FTIR spectroscopy (Wang et al 2000; Zhu et al 2002). They also reported alkyne creation in irradiated PC for electronic stopping power values higher than $3 \cdot 3 \mathrm{MeV} \mathrm{mg}^{-1} \mathrm{~cm}^{2}$. Studies of thermal and structural properties of $62 \mathrm{MeV}$ protons irradiated PC were carried out at different doses and it was reported that thermal stability decreased as dose increased (Mishra et al 2003). The main aim of the present work was to investigate the electrical, thermal and structural properties of $3 \mathrm{MeV}$ proton irradiated PC at different fluences.

\section{Experimental}

Three pieces of MFD (chemical name, polycarbonate; composition, $\left(\mathrm{C}_{16} \mathrm{H}_{14} \mathrm{O}_{3}\right)_{n}$; density, $\left.1.2 \mathrm{~g} / \mathrm{cm}^{3}\right)$ each of thickness $413 \mu \mathrm{m}$ and size $1.5 \times 1.5 \mathrm{~cm}^{2}$ were cut from the sheet available from Good Fellow Corporation (UK). These samples were irradiated by $3 \mathrm{MeV}$ proton beam at the Physics Department, Punjab University, Chandigarh. The irradiation was performed in vacuum $\left(10^{-6}\right.$ Torr $)$ at room temperature. The beam current density was of the order of $30 \mathrm{nA} / \mathrm{cm}^{2}$ and the samples were irradiated at three different fluences of $10^{13}, 10^{14}$ and $10^{15}$ ions $/ \mathrm{cm}^{2}$. The proton beam of diameter $6 \mathrm{~mm}$ was incident perpendicular to the target. To study the structural changes, in- 
cluding the alteration in position and intensity of the characteristic bands, the FTIR spectra of all samples were recorded in the wave number range $4000-500 \mathrm{~cm}^{-1}$ (Bomem Canada, Model-104) with a resolution of $4 \mathrm{~cm}^{-1}$. The electrical properties of all samples were studied after irradiation. The resistance, dielectric loss ( $\tan \delta$ ) and capacitance measurements were carried out using an LCR meter (Hewlett Packard 4284 A) over the frequency range $100 \mathrm{~Hz}-1 \mathrm{MHz}$ at room temperature. The a.c. conductivity was calculated using the relation,

$$
\sigma=\left(2 \pi f C_{\mathrm{p}} D t\right) \mathrm{A}^{-1}(\Omega \cdot \mathrm{cm})^{-1} .
$$

The dielectric constant was calculated using the relation,

$$
\varepsilon=C_{\mathrm{p}} / C_{0},
$$

where $C_{\mathrm{p}}$ is the capacitance measured using an LCR meter, $f$ the frequency, $D$ the dielectric loss and $C_{0}=\varepsilon_{0} A / t$, where $\varepsilon_{0}$ is the permittivity of vacuum and $A$ and $t$ are cross-sectional area of the electrode and thickness of the sample, respectively. The electrical contact was made by applying an air drying type silver paste (i.e. metalpolymer-metal structure). The thermogravimetric analysis (TGA) was recorded using a SIEKO thermal analysis (TGA-220) system in the presence of air from room temperature to $550^{\circ} \mathrm{C}$ at a predetermined heating rate of $10^{\circ} \mathrm{C} / \mathrm{min}$. Differential scanning calorimetry (DSC) measurement was carried out with a SIEKO calorimeter (DSC-220) calibrated through the melting points of indium and tin. About $10 \mathrm{mg}$ of pristine as well as irradiated samples were scanned in the temperature range 40 $325^{\circ} \mathrm{C}$ at a predetermined heating rate of $10^{\circ} \mathrm{C} / \mathrm{min}$.

\section{Results and discussion}

The electronic $(\mathrm{d} E / \mathrm{d} x)_{\mathrm{e}}$ and the nuclear $(\mathrm{d} E / \mathrm{d} x)_{\mathrm{n}}$ stopping power profiles for $\mathrm{MeV}$ ions transiting polymer material show that $(\mathrm{d} E / \mathrm{d} x)_{\mathrm{e}}$ dominates at shallower penetration depths compared with $(\mathrm{d} E / \mathrm{d} x)_{\mathrm{n}}$ which is maximum at the end of the ion track. The projected range, electronic and nuclear stopping power were calculated using SRIM2000 (Ziegler 2000). The projected range was calculated to be $126 \mu \mathrm{m}$, which is $3 \cdot 2$ times less than the thickness of the MFD film. The electronic stopping and nuclear stopping powers were calculated to be $1.13 \times 10^{-1} \mathrm{eV} / \AA$ and $6.9 \times 10^{-5} \mathrm{eV} / \AA$, respectively.

\subsection{FTIR spectral analysis}

The FTIR spectra of the pristine and irradiated samples are shown in figure 1 . The absorption bands as obtained from the pristine spectrum are identified as: (A) $765 \mathrm{~cm}^{-1}$ : out of phase skeletal vibration of $\mathrm{C}-\mathrm{H}$ deformation; (B) $1030 \mathrm{~cm}^{-1}: \mathrm{C}-\mathrm{O}$ stretching vibration; (C) $1645 \mathrm{~cm}^{-1}$ : $\mathrm{C}=\mathrm{C}$ phenyl ring stretching vibration; (D) $1775 \mathrm{~cm}^{-1}: \mathrm{C}=\mathrm{O}$ stretching vibration; (E) $2594 \mathrm{~cm}^{-1}$ : hydroxyl stretching bond; (F) $2968 \mathrm{~cm}^{-1}: \mathrm{CH}_{3}$ stretching vibration; and (G) $3060 \mathrm{~cm}^{-1}$ : $\mathrm{C}-\mathrm{H}$ stretching vibration of aromatic compounds. It is observed that there is no change in overall structure of the polymer but minor changes in intensities were observed up to the fluence of $10^{14}$ ions $/ \mathrm{cm}^{2}$. The minor changes in the peaks of the irradiated samples may be due to the breakage of few bonds in the ladder structure, but this will not change the overall structure of the polymer. The spectrum corresponding to $10^{15}$ ions $/ \mathrm{cm}^{2}$ revealed that the material suffered severe degradation through bond breakage and a significant change in the structure of the polymer.

\subsection{A.c. electrical frequency response}

Figure 2 shows the variation of electrical conductivity with $\log$ of frequency for the pristine and irradiated samples. A sharp increase in conductivity was observed around $300 \mathrm{kHz}$ for pristine and irradiated samples. It is also observed that the conductivity increases as fluence increases. The increase in conductivity due to irradiation may be attributed to scissioning of the polymer chains, resulting in an increase of free radicals, unsaturation, etc. An a.c. field of sufficiently high frequency applied to a metal-polymer-metal structure may cause a net polarization, which is out of phase with the field. This results in a.c. conductivity; it appears at frequencies greater than that at which traps are filled or emptied (Jonscher 1977; Singh et al 2004).

Figure 3 shows a plot of dielectric constant $(\varepsilon)$ vs $\log F$ at ambient temperature for pristine and irradiated MFD

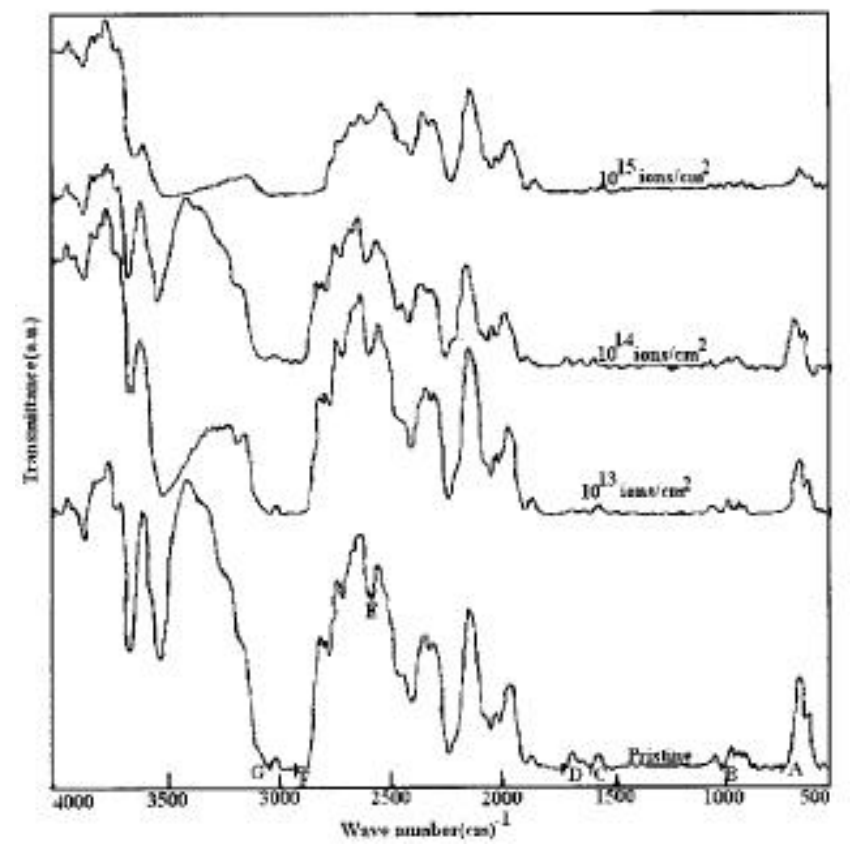

Figure 1. FTIR spectra of pristine and irradiated MFD. 
samples. As evident from the graph, the dielectric constants remain almost constant up to $100 \mathrm{kHz}$ and then decreases at higher frequencies. At lower frequencies the motion of the free charge carriers is constant and thus the dielectric constant is constant. As the frequency increases, the charge carriers migrate through the dielectric and get trapped against a defect site and induce an opposite charge in its vicinity, as a result of which motion of charge carriers is slowed down and the value of dielectric constant decreases.

Figure 4 represents a plot of loss factor $(\tan \delta)$ vs $\log$ frequency for pristine and irradiated MFD films at ambient temperature. The loss factor $(\tan \delta)$ decreases expo-

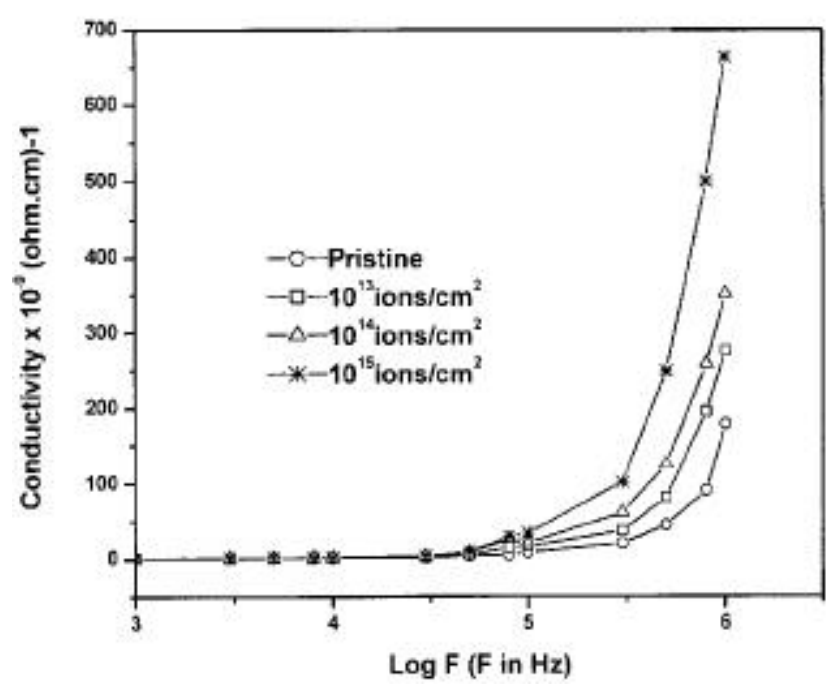

Figure 2. A.c. conductivity vs log frequency plot for pristine and irradiated MFD.

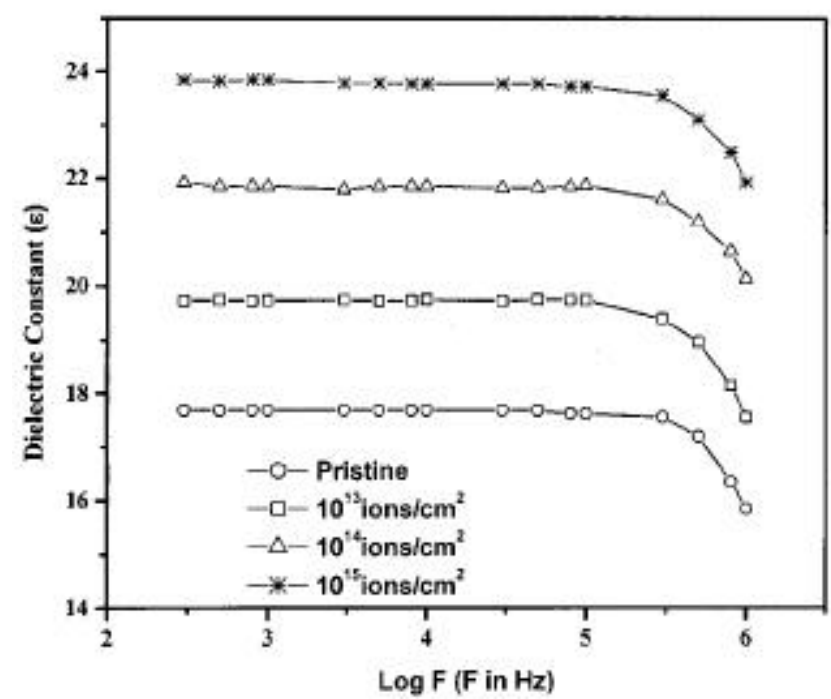

Figure 3. Plot of dielectric constant vs log frequency for pristine and irradiated MFD. nentially as frequency increases. It is also observed that the loss factor increases as fluence increases. The increase in loss factor with fluence may be due to scissioning of polymer chains, resulting in an increase of free radicals, etc. The $\tan \delta$ has a positive value, indicating the dominance of inductive behaviour.

3.2a TGA analysis: As depicted in figure 5, the pristine and irradiated (at the fluence of $10^{14}$ ions $/ \mathrm{cm}^{2}$ ) samples are thermally stable up to $313^{\circ} \mathrm{C}$ and $296^{\circ} \mathrm{C}$, respectively. On the other hand, the stability of irradiated sample (at the fluence of $10^{15}$ ions $/ \mathrm{cm}^{2}$ ) only remained up to $255^{\circ} \mathrm{C}$. This change clearly indicates that up to the fluence of

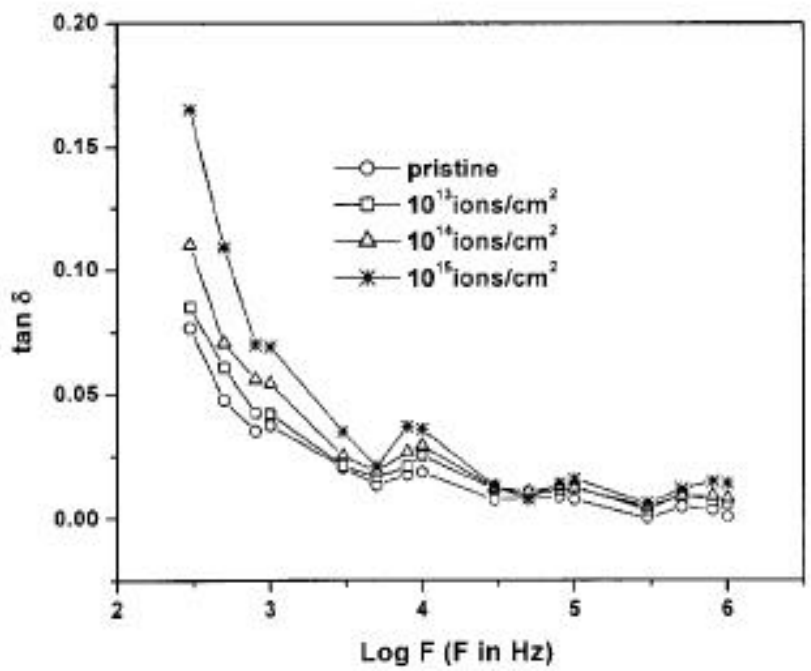

Figure 4. Variation of $\tan \delta$ vs $\log$ frequency for pristine and irradiated MFD.

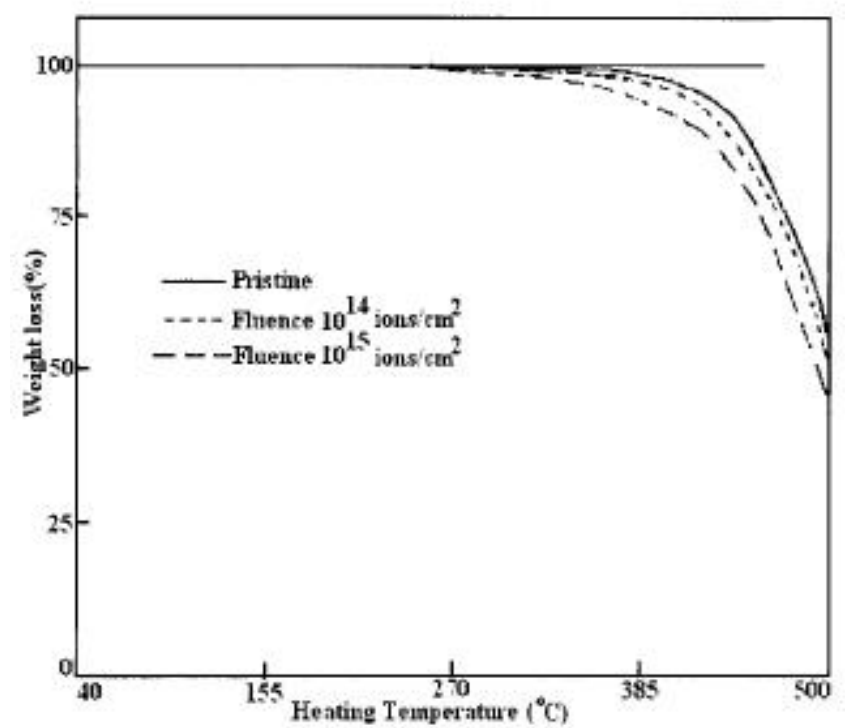

Figure 5. Decrease in thermal stability with fluence as shown in TGA thermogram. 


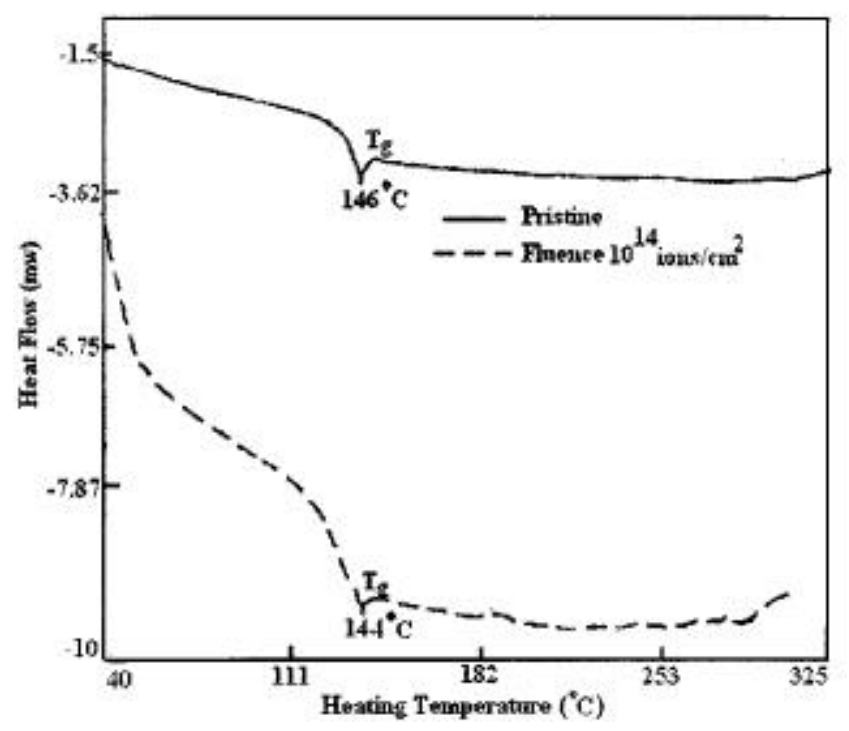

Figure 6. Decrease in $T_{\mathrm{g}}$ with fluence $\left(10^{14} \mathrm{ions} / \mathrm{cm}^{2}\right)$ as shown in DSC thermogram.

$10^{14} \mathrm{ions} / \mathrm{cm}^{2}$, the system remains reasonably organized but becomes quite disorganized when the fluence of $10^{15}$ ions $/ \mathrm{cm}^{2}$ is used and thermal stability reduced by about $19 \%$. Some bond formation i.e. formation of a more organized structure, seems to be happening up to the fluence of $10^{14} \mathrm{ions} / \mathrm{cm}^{2}$. At the limiting heating temperature of $500^{\circ} \mathrm{C}$, the weight loss of pristine and irradiated MFD was about $46 \%, 48 \%$ and $59 \%$, respectively. From the data, it is evident that no significant change was observed up to the fluence of $10^{14}$ ions $/ \mathrm{cm}^{2}$, which is also shown by FTIR spectroscopy (figure 1).

3.2b DSC analysis: Figure 6 shows the DSC thermograms for pristine and irradiated samples. The glass transition temperature $\left(T_{\mathrm{g}}\right)$ appeared around $146^{\circ} \mathrm{C}$ and no endothermic melting $\left(T_{\mathrm{m}}\right)$ peak was observed in the pristine sample. By increasing the ion fluence up to $10^{14}$ ions $/ \mathrm{cm}^{2}$, the $T_{\mathrm{g}}$ was shifted to lower temperature (i.e. $144^{\circ} \mathrm{C}$ ). The minor change observed due to the crosslinking and scissioning of the irradiated MFD is also evidenced from TGA thermogram and FTIR spectrum at the fluence of $10^{14}$ ions $/ \mathrm{cm}^{2}$. The decrease in $T_{\mathrm{g}}$ with further increase in proton fluence support the fact that chain scission predominates by irradiation.

\section{Conclusions}

The FTIR spectra indicate that MFD is chemically degraded at the highest proton fluence used, viz. $10^{15}$ ions/ $\mathrm{cm}^{2}$. On the other hand, there was no significant change in the absorbance bonds of MFD observed by proton irradiation up to the fluence of $10^{14}$ ions $/ \mathrm{cm}^{2}$. The minor changes in the peaks of irradiated samples may be due to breakage of a few bonds in the ladder structure, but this will not change the overall structure of the polymer. The increase in dielectric properties due to irradiation may be attributed to scissioning of polymer chains, resulting in an increase of free radicals, unsaturation etc. The decrease in thermal stability at the highest proton fluence $\left(10^{15}\right.$ ions $\left./ \mathrm{cm}^{2}\right)$ indicates that the polymer underwent chain-scission by proton irradiation. No significant change in the position of $T_{\mathrm{g}}$ was observed for pristine and irradiated samples at the fluence of $10^{14}$ ions $/ \mathrm{cm}^{2}$, which is also corroborated with TGA thermogram and FTIR spectra at the fluence of $10^{14}$ ions $/ \mathrm{cm}^{2}$.

\section{Acknowledgements}

The authors are thankful to the operating staff of Cyclotron, Department of Physics, Panjab University, Chandigarh and to the Head, Department of Physics, S.P. University, Vallabh Vidyanagar, for providing laboratory facilities and ABS Bayer, Moxi for providing thermal analysis facilities. Partial financial support given by NSC, New Delhi, is gratefully acknowledged.

\section{References}

Chipara M I and Reyes-Romero J 2002 Nucl. Instrum. \& Meth. B185 77

Dehaye F, Balanzat E, Ferain E and Legras R 2003 Nucl. Instrum. \& Meth. B209 103

Ferain E and Legras R 1993 Nucl. Instrum. \& Meth. B82 539

Jerome C, Demoustier-Champagne S, Legras R and Jerome R 2000 Chem. Eur. J. 63089

Jonscher A K 1977 Nature 267673

Mishra R, Tripathy S P, Dwivedi K K, Khathing D T, Ghosh S and Fink D 2003 Rad. Meas. 36719

Piraux L, Dubois S, Duvail J L, Radulescu A, DemoustierChampagne S, Ferain E and Legras R 1999 J. Mater. Res. 14 3042

Singh N L, Sharma Anita, Shrinet V, Rakshit A K and Avasthi D K 2004 Bull. Mater. Sci. 27227

Steckenreiter T, Balnzat E, Fuess H and Trautmann C 1999 Nucl. Instrum. \& Meth. B151 161

Venkatesan T, Calcagno L, Elman B S and Foti G 1987 Ion beam modification of insulators (eds) P Mazzoldi and G W Arnold (Amsterdam: Elsevier) Vol. 2, p. 301

Wang Y et al 2000 Nucl. Instrum. \& Meth. B164-165 420

Ziegler J F 2000 The stopping range of ions in matter (SRIM) (New York: IBM Research) p. 1

Zhu Z, Sun Y, Liu C, Liu J and Jin Y 2002 Nucl. Instrum. \& Meth. B193 271 